\title{
Miranda
}

Revue pluridisciplinaire du monde anglophone /

Multidisciplinary peer-reviewed journal on the English-

speaking world

$20 \mid 2020$

Staging American Nights

\section{Notre Top 5 des films anglophones de 2019}

\section{David Roche et Vincent Souladié}

\section{(2) OpenEdition}

\section{Journals}

Édition électronique

URL : http://journals.openedition.org/miranda/26112

DOI : $10.4000 /$ miranda.26112

ISSN : 2108-6559

\section{Éditeur}

Université Toulouse - Jean Jaurès

\section{Référence électronique}

David Roche et Vincent Souladié, « Notre Top 5 des films anglophones de 2019 », Miranda [En ligne], 20 | 2020, mis en ligne le 30 mars 2020, consulté le 16 février 2021. URL : http://

journals.openedition.org/miranda/26112 ; DOI : https://doi.org/10.4000/miranda.26112

Ce document a été généré automatiquement le 16 février 2021.

\section{c) (i)}

Miranda is licensed under a Creative Commons Attribution-NonCommercial-NoDerivatives 4.0 International License. 


\title{
Notre Top 5 des films anglophones de 2019
}

\author{
David Roche et Vincent Souladié
}

2019 aura surtout été l'année du cinéma non anglophone, avec quelques très grands films dont Portrait de la jeune fille en feu (France), quatrième long métrage de Céline Sciamma qui mêle merveilleusement féminisme, poésie et réflexivité, Douleur et gloire/ Dolor y gloria (Espagne) le nouveau métafilm de Pedro Almodóvar, et surtout le triomphe de Parasite (Corée du Sud, Bong Joon Ho) à Cannes et - plus surprenant et sans doute politique - aux Oscars, avec ce premier Oscar du Meilleur Film attribué à un film étranger. Néanmoins le cinéma notamment américain a également connu une bonne année. Les franchises Avengers et Star Wars se sont conclues sur une note tout à fait honorable, avec Avengers : End Game (USA, les frères Russo) et surtout Star Wars IX : The Rise of Skywalker (USA, J. J. Abrams) dont la révélation finale concernant Rey évite la faute idéologique commise dans Games of Thrones (HBO, 2011-2019) à travers le thème de la filiation (la parenté de Jon Snow et de Daenerys Targaryen). De l'autre côté du spectre, le cinéma indépendant aura eu sa découverte de l'année avec The Last Black Man in San Francisco (USA), film dystopique sur le phénomène de la gentrification, écrit et réalisé avec un formalisme assumé par le duo Joe Talbot et Jimmie Falls qui tient aussi le premier rôle. Retours solides pour : Greta Gerwig dont la nouvelle adaptation de Little Women/ Les Quatre filles du docteur Marsh (USA) fait ressortir la noirceur de l'œuvre de Louisa May Alcott ; James Gray dont le plastiquement somptueux Ad Astra (Chine/USA) fait parfois oublier un scénario prévisible, parfois absurde et fadasse après ces récentes réussites que sont Interstellar (USA/UK/Canada, Christophe Nolan, 2014) et surtout Arrival/Premier contact (USA/Canada, Denis Villeneuve, 2016) ; Ryan Johnson qui offre un whodunit efficace et divertissant avec Knives Out/ À couteaux tirés (USA); ou encore James Mangold dont le biopic sportif (et Oscar-bait raté) Ford v. Ferrari/ Le Mans 66 (USA/France) permet de passer un bon moment en compagnie du toujours sympathique Matt Damon et du hargneux Christian Bale ; Sam Mendes qui, avec 1917 (USA/UK/India/Spain/Canada), dont la somptueuse scène nocturne des ruines de guerre sublime un film agréable mais somme toute assez convenu, nous démontre, à l'inverse d'un Steven Spielberg avec Saving Private Ryan/Il faut sauver le soldat Ryan (USA, 
1998) ou Alejandro G. Iñárritu avec Birdman (USA, 2014), que la prouesse technique (le plan-séquence) peut facilement prendre le dessus sur le récit et la dramaturgie; les frères Safdie, avec leur sixième long métrage Uncut Gems (USA), font en quelque sorte la démonstration inverse, la technologie de mise au point automatisée Light Ranger 2 permettant une grande liberté de mouvement à l'acteur Adam Sandler qui galvanise une narration centrée sur un anti-héros dont la chute inexorable rappelle aussi bien Fargo (USA/UK, les frères Coen, 1996) que la trilogie Pusher de Nicolas Winding Refn (Danemark, 1996-2005); et enfin Taika Waititi qui revient à la figure de l'enfant, qui était au cœur de Boy (Nouvelle-Zélande, 2010) et de l'excellent Hunt for the Wilderpeople (Nouvelle-Zélande, 2016), avec cette adaptation (Nouvelle-Zélande/République Czec/ USA) du roman satirique de Christine Leunens. Plus notables, les seconds films de Jordan Peele, de Robert Eggers et d'Ari Aster ont confirmé avec brio le renouveau du cinéma d'horreur américain : qu'il s'agisse de la satire politique proposée dans Us (USA/ Chine/Japon) qui déplace l'accent sur la question raciale à la question sociale; du surréalisme atmosphérique et hyper-formaliste de The Lighthouse (Canada/USA) avec ses influences à la littérature américaine du XIXe (Herman Melville, Edgar Poe) et au cinéma muet européen des années 1920 (Jean Epstein, F. W. Murnau); ou de l'allégorie du deuil offerte Midsommar (USA/Suède), variation sur le thème du Wicker Man (UK, Robin Hardy, 1973) qui relève le défi de l'horreur en plein jour. Leurs pairs ont aussi proposé des films solides: Woody Allen, avec l'agréable A Rainy Day in New York (USA), qui permet à Timothy Chalomet de camper Gastby, personnage allenien naif qui doit plus à J. D. Sallinger qu'à Fitzgerald, et une charmante Elle Fanning qui - de manière plus suspecte à l'ère \#MeToo - semble en partie dédouaner les prédateurs sexuels incarnés par Jude Law, Diego Luna et Liv Schrieber ; le tandem Ken Loach-Paul Laverty, qui clôt son œuvre avec Sorry We Missed You (UK/France/Belgique), dont le pessimisme n'augure rien de bon pour la Grande-Bretagne post-Brexit; Martin Scorsese avec la production Netflix, The Irishman (USA), qui, au-delà du rajeunissement numérique dont on a tant parlé, permet à Robert DeNiro, à Al Pacino et surtout un Joe Pesci apaisé de jouer dans un autre registre que dans les films de gangsters qui ont fait leur gloire. Moins convaincants : The Mountain : une odyssée américaine (USA, Rick Alverson) et The Souvenir (UK/USA, Joanna Hogg), dans lesquels des castings de choc (Tye Sheridan et Jeff Goldblum dans le premier, Honor Swinton Byrne et Tom Burke dans le second) tentent en vain de lutter contre un formalisme glacial pour faire passer une émotion.

\section{A Hidden Life (USA/UK/Allemagne, Terrence Malick)}

2 Il était annoncé depuis longtemps que Terrence Malick revenait enfin avec A Hidden Life à la forme narrative de ses débuts, comme si son virage esthétique vers un cinéma de plus en plus dysnarratif depuis Tree of Life (2011) ne pouvait représenter qu'une parenthèse appelée à se refermer. Comme cela est toujours le cas avec Malick, le film déjoue en partie ces attentes et ne saurait se prévaloir d'une facture dramatique classique. Après une trilogie contemporaine autobiographique (To the Wonder [2012], Knight of Cups [2015], Song to Song [2017]), Malick revient surtout au film d'époque délocalisé puisque A Hidden Life relate l'histoire vraie de Franz Jägerstätter (August Diehl, dans un rôle diamétralement opposé à celui qu'il tenait en 2009 dans Inglourious Basterds), paysan autrichien emprisonné, persécuté, et finalement exécuté pour avoir catégoriquement refusé de porter l'uniforme et de servir l'Allemagne d'Hitler, quitte à ne pas signer le serment d'allégeance qui aurait pu in fine lui sauver la vie. Il est aisé de 
comprendre ce qui a poussé Malick à s'intéresser à la vie de cet objecteur de conscience béatifié par Benoit XVI en 2007, lui qui assume de plus en plus ouvertement dans ses films son héritage catholique et les questionnements de la foi (il a achevé fin 2019 une vie de Jésus Christ tournée en Jordanie, The Last Planet). On retrouve également dans le film certains thèmes chers au cinéaste : l'exil et la quête du paradis perdu, la pureté désintéressée de l'amour contre l'oppression du système, l'immuabilité de la nature contre le fracas absurde et passager des hommes. Au fond, ce ne sont pas forcément ces questions personnelles ni ces thématiques qui rendent fascinant le cinéma de Malick mais la manière dont il réadapte son langage formel. L'auteur tire grand profit des expérimentations formalistes élaborées dans ses quatre derniers films en les mettant ici au service d'une histoire et, surtout, en leur donnant une portée politique. Mixer les régimes d'images (les plans filmés avec un appareil photo numérique montés bout à bout avec des plans tournés en caméra HD dans To the Wonder, par exemple) lui permet ici de faire cohabiter deux mondes hétérogènes, irréels l'un à l'autre : le sacre de Hitler (dans des plans extraits du Triomphe de la Volonté de Leni Riefensthal, 1935) et la nature aveugle occupée par les paysans. Les plans d'intérieur cadrés en grand angle déformant matérialisent, avec un sens du grotesque kubrickien que l'on ne connaissait pas à Malick, l'aliénation du pouvoir dictatorial (les bottes géantes de Bruno Ganz, le Hitler de La Chute [Allemagne/Autriche/Italie, Oliver Hirschbiegel, 2004]). Il a été reproché à Malick l'usage de l'allemand non sous-titré pour faire vociférer les habitants tentés par les promesses du Troisième Reich, ce qui serait le signe de sa part d'une représentation caricaturale et manichéenne de l'Histoire, adaptée à un public américain. C'est faire là un étrange procès à ce spécialiste d'Heidegger, traducteur de son œuvre philosophique aux États-Unis. La barrière instaurée par ce procédé n'est pas idiomatique, en réalité, mais existentielle. Du point de vue de l'éthique rigoriste de Jägerstätter, certaines idées et certains discours doivent seulement rester à distance morale, dans une zone noire indéchiffrable.

\section{Joker (USA/Canada, Todd Phillips)}

3 Joker fut le film américain le plus remarqué de l'année, et celui dont le succès fut le plus controversé, de son triomphe au festival de Venise aux nombreux commentaires sur l'ambiguïté de son discours politique. Pour les uns, il s'agit du pari réussi d'un superhero movie qui, tout en reprenant le parcours génétique, prend le risque de se détourner radicalement de nombreux codes du genre pour s'aventurer sur le terrain du naturalisme social. Pour les autres, il s'agit du succès de circonstance d'une œuvre faussement provocatrice surfant sur l'air du temps; ces réserves sont comparables à celles qu'avait suscité Fight Club (USA/Allemagne, David Fincher, 1999) en son temps : film produit par le système et prônant l'effondrement de celui-ci. C'est bien sûr cette ambiguïté qui rend Joker pertinent, et correspond d'ailleurs au parcours dramatique de son personnage éponyme, porté aux nues contre son gré comme une figure révolutionnaire pour avoir voulu attirer l'attention des médias (on y aura vu des échos de Occupy Wall Street aux US ou des Gillets Jaunes en France). Puisque le Joker encourage désormais les performances actor's studio, le rôle ne pouvait qu'être taillé sur-mesure pour Joachin Phoenix, dont l'énergie inquiétante mais fragile lui permet de se démarquer subtilement de l'interprétation nihiliste d'Heath Ledger dans The Dark Knight (USA/UK, Christopher Nolan, 2008) autant que du Grand Guignol de Jack Nicholson dans Batman (USA, Tim Burton, 1989). Si le film de Philips fait de Thomas 
Wayne un entrepreneur mégalomane convaincu qu'il a l'étoffe d'un politique, il pourrait être un prolongement en mode mineur des opus de Nolan, dont il ne se détourne pas complètement. Comme Nolan l'envisageait au départ de sa trilogie, Philips cherche à retrouver une ambiance urbaine des années 70's-80's qui passe par des références ouvertes à Death Wish (USA, Michael Winner, 1974), à Taxi Driver (USA, Martin Scorsese, 1976) ou à The King of Comedy (USA, Martin Scorsese, 1983), renforcé bien sûr par la présence de Robert DeNiro qui cède sa place à son digne héritier Joaquim Phoenix pour reprendre un rôle équivalent à celui de Jerry Lewis dans le film de 1983. De fait, et même si le film se retient comparé à ses modèles, la violence et la noirceur nihiliste surprennent. Sur un plan esthétique, la richesse sonore et chromatique de Joker n'est pas pour autant déphasée par rapport à son temps. Cette expérience d'une odyssée psychologique et sensible dans un décor urbain âpre et fiévreux est à rapprocher dans une certaine mesure des récents films des frères Safdie (Good Time [2017], Uncut Gems [2020]), mais aussi de You Were Never Really Here/A Beautiful Day (UK/France/USA, Lynne Ramsay, 2017), dans lequel Phoenix apparaissait déjà, sous des traits et une silhouette bien différents. Son statut de satire allégorique rend Joker plus accessible que ces œuvres-là, mais il n'en reste pas moins le chef de file de cette noirceur montante du cinéma américain contemporain, de plus en plus palpable.

\section{Luce (USA, Julius Onah)}

Il est des films qui marquent avant tout par la qualité de leur scénario et de leur interprétation. C'est le cas cette année de Marriage Story. C'est aussi le cas de ce premier long métrage, collaboration entre le réalisateur afro-américain Julius Onah et le scénariste J.C. Lee qui adapte ici sa propre pièce produite à New York en 2013. Aux côtés de Tim Roth, d'Octavia Spencer et de Naomi Watts - tous excellents ! - dans des rôles secondaires (les parents et l'enseignante), le jeune Kelvin Harrison Jr. crève l'écran dans le rôle éponyme d'un enfant congolais adopté par une famille américaine (blanche de classe moyenne-supérieure, l'image même des " white liberals »), devenu la figure de l'excellence (scolaire, sportive, sociale) dans son lycée. Le maitre mot du film est l'« ambiguïté ", ambiguïté qui laisse planer l'ombre d'un doute tout au long du film. Il n'y a pas de bons ou de méchants dans ce mélodrame aux allures de thriller psychologique : il y a des points de vue qui entrent en concurrence, qui se contredisent et parfois même qui sont tous deux « vrais » ou " faux » en même temps. Est ainsi posée la relation complexe entre la subjectivité humaine et une conception intersectionnelle de l'identité, démontrant que la portée de celle-ci ne doit pas se limiter à une conception juridique, voire même politique au sens large. Cette ambiguïté est habilement exprimée à travers les ressorts habituels du thriller (ombres portées, montage elliptique), mais c'est avant tout sur le visage et dans la voix de Harrison Jr que tout se joue: l'angélique Luce affiche confiance, intelligence, maîtrise mais est régulièrement parcourue par des ondes émotionnelles traduisant une incertitude et une vulnérabilité authentique ou feinte (là est la question). La révélation tarde et quand elle arrive, elle ne fait que renforcer le sentiment d'incertitude. La réussite de Luce tient avant tout à l'intelligence avec laquelle elle fait de la question de l'identité un enjeu sensible et donc éminemment esthétique. 


\section{Marriage Story (UK/USA, Noah Baumbach)}

5 Alors que Woody Allen revenait cette année à une forme de légèreté screwball avec le très enlevé A Rainy Day In New York, c'est l'un de ses héritiers directs, qui réitère le genre des drames de chambre familiaux new-yorkais doux-amers, comme il s'y était d'ailleurs essayé dès son tout premier film Les Berkman se séparent/The Squid and the Whale (USA, 2005), qui était déjà la chronique du divorce d'un couple bourgeois. La rupture est d'ailleurs sous-tendue par un autre duel, celui-ci plus culturel, entre New York et Los Angeles, le théâtre et l'industrie cinématographique et télévisuelle. Noah Baumbach trouve chez Allen des thèmes qu'il traite toutefois avec une authenticité plus terre à terre. Le ton de Marriage Story pourrait aussi rappeler de prime abord la simplicité mélodramatique de Kramer vs. Kramer (Robert Benton, 1979) mais le propos s'avère en fin de compte plus ambitieux et subtil. Le film met tout d'abord à l'honneur deux des meilleurs comédiens de leur génération, capable d'alterner les blockbusters et les films d'auteurs indépendants sans décevoir et sans brouiller leur image auprès du public. Si l'on savait depuis le début de leur carrière que Scarlett Johansson et Adam Driver étaient capables de jouer les âmes fragiles avec autant de naturel, il y a bien longtemps - depuis Paterson (USA, Jim Jarmusch, 2016) pour Driver et Under the Skin (UK/USA/Suisse, Jonathan Glazer, 2014) pour Johansson - qu'ils n'avaient pas été servis par des rôles aussi bien écrits, et par une mise en scène pensée pour donner du temps et de l'amplitude à leur jeu. L'inflexion de la voix, le détail d'un regard ou d'un geste, l'interaction des corps nourrissent le sentiment du vécu de cette expérience amoureuse et maritale en partie autobiographique. Via l'importance de l'écrit et de la parole, les racines littéraires et théâtrales de la méthode employée par Baumbach sont mises en abyme dans le film, lequel se veut l'auscultation détaillée d'une rupture et de sa mise en fiction. Le sujet sous-jacent pourrait alors être celui-ci, par ailleurs délivré par le titre même du film : toute rupture naissant de la collision de deux points de vue adverses donne forcément lieu à un récit fictionnel. C'est alors que Marriage Story épate là où on ne l'attendait pas: dans le portrait des avocats tout aussi cyniques qu'humanistes des deux époux opposés (Laura Dern, Ray Liotta, Alan Alda), orateurs et narrateurs apocryphes de la déliquescence du mariage, exagérant ou dénaturant les événements vécus dans l'intérêt de leurs clients, ou de leur propre ego. Baumbach affirme que de la rencontre à la rupture, l'équilibre d'une relation entre deux êtres repose sur une négociation fictionnelle avec les faits, sur une histoire sur laquelle les deux s'accordent. Marriage Story raconte l'histoire de cette fiction tacite.

\section{Once Upon a Time ... in Hollywood (USA/UK/Chine, Quentin Tarantino)}

Sans doute son meilleur film depuis Inglourious Basterds (Allemagne/USA, 2009) et l'une des plus grandes réussites de 2019, le neuvième long métrage du scénariste-réalisateur américain est à la fois un film-somme qui, situé dans le Los Angeles de 1969, poursuit la démarche uchronique des trois précédents films tout en revisitant les lieux de ses trois premiers films. Les clins d'œil aux films précédents (surtout à Inglourious Basterds) et aux influences habituelles (Leone, Corbucci et le Western italien) se multiplient et se voient même intégrés aux récits, Rick Dalton relançant sa carrière grâce au « deuxième plus grand réalisateur de Westerns spaghettis »! Un air de déjà vu donc, renforcé par la 
présence de Leonardo DiCaprio et de Brad Pitt, de Robert Richardson à la photographie et d'une bande-son impeccable. Et pourtant l'inattendu émerge à travers un ton à la fois tendre et mélancolique que l'on avait entrevu principalement dans Jackie Brown (USA, 1997). Après une introduction énergique et hautement parodique (à l'image du début de carrière de Rick Dalton), la narration relâche le rythme - et c'est là que l'influence de Rio Bravo (USA, Howard Hawks, 1959) se fait sentir - pour nous laisser le temps de naviguer les trajectoires et de nous imprégner de la présence de ses personnages (Rick Dalton, Cliff Booth et Sharon Tate) et de la cité des anges. On retrouve la critique de la masculinité blanche au cœur de Reservoir Dogs, incarnée dans des personnages machos, xénophobes et étroits d'esprit, tout en encourageant le pathos pour la chute de Rick (mention spéciale pour la scène dans laquelle une jeune actrice lui fait prendre conscience que son destin ressemble en tout point à celui du héros westernien dont il lit le récit) et de la sympathie pour la fidélité sans faille de cliff (à l'image de son chien Brandy). La fin du film est poignante non seulement parce qu'on est heureux que Sharon Tate soit sauvée (à ce stade c'était attendu, on se demandait juste comment), mais aussi et surtout parce que Cliff est bel et bien un «bon ami » malgré le narcissisme de Rick et la barrière socioprofessionnelle qui les sépare et que les longs trajets en voiture de Booth ne cessent de souligner. Tarantino en profite aussi pour à la fois célébrer le Polanski qui a réalisé Rosemary's Baby (USA, 1968) et le comparer implicitement et défavorablement à Cliff dont le comportement quand il refuse les avances d'une mineure (Pussycat, force vitale au même titre que Tate) tend plutôt à démentir les rumeurs mais dont la situation en bas de la hiérarchie de l'industrie cinématographique ne lui a jamais permis de se remettre. Quant à la reconstitution fétichiste du Los Angeles de 1969, démarche qui peut paraître à première vue paradoxale pour un réalisateur qui dit s'inspirer plus du cinéma que de la vie, elle nous (dé) montre splendidement combien est ténue la frontière entre la ville cinématographique et la ville réelle dont le film rend fidèlement compte de la dimension socioethnique (la présence latino) de sa topographie. Le tragique tient avant tout de ce que le film est structuré autour de la chronique de la mort annoncée de Sharon Tate, figure iconique que le film va d'abord transformer en force symbolique de la vie (les scènes de danse) mais que la brillance de Margot Robbie finit par humaniser à travers une série de gestes quotidiens (un réveil, la prise en stop d'une autostoppeuse) et surtout par une virée au cinéma pour se contempler sur l'écran. Cette scène qui célèbre les plaisirs du cinéma et de sa création fait de Tate la double de Tarantino, qui s'est lui aussi délecté à assister de multiples fois à des projections de Reservoir Dogs. Si Rick incarne l'incertitude que peut ressentir tout artiste et Cliff un impossible idéal de sérénité (malgré son vécu), c'est Tate qui est le cœur du film et le cœur de Tarantino. Once Upon a Time... in Hollywood n'est pas une ode au classicisme hollywoodien - il est d'ailleurs étonnant que personne n'ait relevé que l'agent qui relance la carrière de Rick est interprété par Al Pacino, l'une des stars du New Hollywood. Contrairement à ce qu'on a pu lire, c'est une ode au cinéma et à la ville. 
INDEX

Thèmes : Film, TV, Video

Keywords : American cinema, British cinema, Canadian cinema, European cinema, New Zealand cinema, independent cinema, blockbuster, art cinema, Une Vie cachée, Joker, Luce, Marriage Story, Once Upon a Time... in Hollywood

Mots-clés : cinéma américain, cinéma britannique, cinéma européen, cinéma néo-zélandais, cinéma indépendant, blockbuster, cinéma d'art, Une Vie cachée, Joker, Luce, Marriage Story, Once Upon a Time... in Hollywood

\section{AUTEURS}

\section{DAVID ROCHE}

Professeur d'études cinématographiques et audiovisuelles

Université Paul Valéry Montpellier 3

david.roche@univ-montp3.fr

\section{VINCENT SOULADIÉ}

Maître de conférences en études cinématographiques et audiovisuelles Université Toulouse Jean Jaurès

vincent.souladie@univ-tlse2.fr 